masses occupied the posterior fossa. (2) The stem of the internal carotid atery was involved by the lesion, therefore, it was possible to demonstrate deviation and obliteration of the artery by carotid angiography. Carotid obliteration migh be one of the possible complications that interfere the clinical picture of atypical acoustic neurinoma. Complete removal of masses in the posterior fossa does not result, therefore, in the improvement of clinical manifestations.

\title{
79. Clinicopathological Study on Cerebral Hemorrhage in Metastatic Foci of Lung Cancer
}

\author{
Toyozo Alzawa, Yuichiro Goto, Goro Araki, \\ Keiichi MuraKami and Nobuhisa Sato \\ Department of Internal Medicine, School of Medicine, Keio University
}

113 cases of lung cancer were autopsied at the Pathological Department of Keio University School of Medicine from 1947 to 1958.30 cases of them had brain metastasis and macroscopic cerebral hemorrhage complicated in about $50 \%$.

Around the metastatic foci with hemorrhage, vascular lesions especially angionecrosis were observed. On the other hand, angionecrosis was not found in foci without hemorrhage. By these facts, the relation between cerebral hemorrhage and vascular change may be partially explained, namely, angionecrosis is considered here to be only a secondary change.

We studied also the important problems related to hemorrhage such as the effect of blood pressure, anemia, medication, arteriosclerosis and histopathological findings of metastatic tumor.

\section{Pre- and Post Operative Adrenocortical Function of Brain Tumor Patients}

\author{
Yoshiaki Takeda, Sataro Jitsukawa, Kenzo Matsuoka, \\ Hiroshi IzUmi AND Tohoru UozumI \\ The First Department of Surgery, Osaka University Medical School
}

The adrenocortical function of patients with brain tumor in our clinic was examined by using ACTH-Z-Test. It was found that some cases of tumors of hypophyseal region showed adrenocortical insufficiency and a few cases of tumors in cerebral hemisphere or posterior cranial fossa showed also insufficiency or abnormal responses.

We have experienced some shock cases in this insufficient number of patients, 